\title{
THE GELFAND THEOREM AND ITS CONVERSE FOR KÄHLER MANIFOLDS
}

\author{
O. KOWALSKI AND L. VANHECKE
}

(Communicated by David G. Ebin)

\begin{abstract}
We characterize the locally Hermitian symmetric manifolds among the homogeneous Kähler manifolds $M$ by each of the following properties:

(i) all $A_{0}(M)$-invariant differential operators on $M$ commute $\left(A_{0}(M)\right.$ denotes the identity component of the group of all holomorphic isometries);

(ii) all geodesics are orbits of one-parameter groups of holomorphic isometries.
\end{abstract}

Recently, D'Atri, Dorfmeister, and Zhao Yan da [1] proved the following characterization of symmetric Siegel domains.

THEOREM A. Let $D$ be a homogeneous Siegel domain and $G$ the identity component of the automorphism group of $D$. Then the algebra of $G$-invariant differential operators on $D$ is commutative if and only if $D$ is a symmetric domain.

The "if" part is an easy modification of the well-known Gelfand theorem (see [3]). It still holds in the following more general situation. Let $M$ be a Hermitian symmetric space (thus a homogeneous Kähler manifold), and let $A_{0}(M)$ denote the identity component of the group of all holomorphic isometries of $M$. Then the algebra of $A_{0}(M)$-invariant differential operators on $M$ is commutative.

In this report we prove a converse of the last statement and this gives an essential generalization of Theorem A.

THEOREM 1. Let $(M, g, J)$ be a homogeneous Kähler manifold. If all $A_{0}(M)$ invariant differential operators on $M$ commute, then $M$ is locally Hermitian symmetric.

COROLLARY. Let $M$ be a simply connected homogeneous Kähler manifold. Then the algebra of $A_{0}(M)$-invariant differential operators on $M$ is commutative if and only if $M$ is Hermitian symmetric.

The proof is based on two lemmas. Let

LEMMA 1 [7]. Let $(M, g)$ be a smooth $n$-dimensional Riemannian manifold.

$$
D=\sum_{i_{1}, \ldots, i_{k}=1}^{n} T^{i_{1} \ldots i_{k}} \nabla_{i_{1} \ldots i_{k}}^{k}
$$

Received by the editors June 30, 1986.

1980 Mathematics Subject Classification (1985 Revision). Primary 53C20, 53C30, 53C55.

Key words and phrases. Invariant differential operator, Hermitian symmetric space, homogeneous Kähler manifold. 
be a differential operator whose coefficients are symmetric and which commutes with the Laplacian $\Delta=\sum_{i, j=1}^{n} g^{i j} \nabla_{i j}^{2}$. Then the following tensor identity holds:

$$
\nabla_{i_{1}} T_{i_{2} \ldots i_{k+1}}+\cdots+\nabla_{i_{k+1}} T_{i_{1} \ldots i_{k}}=0 .
$$

A short proof can be found in [4].

LEMMA 2. A Kähler manifold $(M, g, J)$ is locally Hermitian symmetric if and only if

$$
\left(\nabla_{X} R\right)(X, J X, X, J X)=0
$$

holds for each tangent vector $X$.

The identity (2) was first investigated by A. Gray in connection with the theory of 3-symmetric spaces, and the proof of Lemma 2 can be obtained by combining Theorem 4.6 and Corollary 4.4 from [2]. A full direct proof (elementary, but rather long) can be found in two parts in [6] and [8]. Although this lemma proved to be useful in many topics of Kählerian geometry, a really short and simple proof is not yet available.

ProOf of THEOREM 1. Define a 4-valent tensor field $T$ on $M$ by putting

$$
T^{i j k l}=T_{i j k l}=R\left(e_{i}, J e_{j}, e_{k}, J e_{l}\right)+R\left(e_{k}, J e_{j}, e_{i}, J e_{l}\right)+R\left(e_{l}, J e_{j}, e_{k}, J e_{i}\right)
$$

with respect to any orthonormal frame (where $R$ is the curvature tensor). Since $M$ is Kählerian, $T^{i j k l}$ is symmetric. Further, $T$ is $A_{0}(M)$-invariant and hence the differential operator

$$
D=\sum_{i, j, k, l=1}^{n} T^{i j k l} \nabla_{i j k l}^{4}
$$

is also $A_{0}(M)$-invariant. Then $D$ must commute with $\Delta$ and Lemma 1 gives $\left(\nabla_{X} T\right)(X, X, X, X)=0$, i.e. $\left(\nabla_{X} R\right)(X, J X, X, J X)=0$ for each tangent vector $X$. Now we use only Lemma 2 to obtain the result.

NOTE A. The converse of the Gelfand theorem is not true in the real case. For instance, there are many naturally reductive homogeneous Riemannian manifolds for which all $I_{0}(M)$-invariant differential operators commute and which are not locally symmetric. Yet, the following weaker converse still holds (see [4]): Let $(M, g)$ be a homogeneous Riemannian manifold and $I_{0}(M)$ the identity component of the full isometry group of $M$. If all $I_{0}(M)$-invariant differential operators on $M$ commute, then the local geodesic symmetries of $(M, g)$ are volume-preserving.

NOTE B. The following result is related to our Theorem 1.

THEOREM 2. Let $(M, g, J)$ be a homogeneous Kähler manifold all of whose geodesics are orbits of one-parameter groups of holomorphic isometries. Then $(M, g, J)$ is locally Hermitian symmetric.

PROOF. Along any fixed geodesic $\gamma$, the function $R\left(\gamma^{\prime}, J \gamma^{\prime}, \gamma^{\prime}, J \gamma^{\prime}\right)$ is constant. Hence $\gamma^{\prime}\left[R\left(\gamma^{\prime}, J \gamma^{\prime}, \gamma^{\prime}, J \gamma^{\prime}\right)\right]=0$, and because $M$ is Kählerian, we get the condition of Lemma 2.

Again, a real analogue holds in the weaker form (see [5]): Let $(M, g)$ be a homogeneous Riemannian manifold all of whose geodesics are orbits of one-parameter groups of isometries. Then the local geodesic symmetries of $(M, g)$ are volumepreserving. 


\section{REFERENCES}

1. J. E. D'Atri, J. Dorfmeister and Zhao Yan da, The isotropy representation for homogeneous Siegel domains, Pacific J. Math. 120 (1985), 295-326.

2. A. Gray, Riemannian manifolds with geodesic symmetries of order 3, J. Differential Geom. 7 (1972), 343-369.

3. S. Helgason, Differential geometry and symmetric spaces, Academic Press, New York, 1962.

4. O. Kowalski and L. Vanhecke, Opérateurs différentiels invariants et symétries géodésiques préservant le volume, C. R. Acad. Sci. Paris I 296 (1983), 1001-1003.

5. O. Kowalski and L. Vanhecke, A generalization of a theorem on naturally reductive homogeneous spaces, Proc. Amer. Math. Soc. 91 (1984), 433-435.

6. K. Sekigawa and L. Vanhecke, Symplectic geodesic symmetries on Kähler manifolds, Quart. J. Math. Oxford 32 (1986), 95-103.

7. T. Sumitomo, On the commutator of differential operators, Hokkaido Math. J. 1 (1972), 30-42.

8. L. Vanhecke and T. J. Willmore, Interaction of tubes and spheres, Math. Ann. 263 (1983), 31-42.

Faculty of Mathematics and Physics, Charles University, Sokolovskà 83, 18600 PRAHA, CZECHOSLOVAKIA

Department of Mathematics, Katholieke Universiteit Leuven, CelestijNENLAAN 200B, 3030 LEUVEN, BELGIUM 